\title{
Correction to: No prominent toxicity of polyethylene microplastics observed in neonatal mice following intratracheal instillation to dams during gestational and neonatal period
}

\author{
YoungHoon Han ${ }^{1} \cdot$ YoungMin Song ${ }^{1} \cdot$ Geun Woo Kim ${ }^{1} \cdot$ ChangSu Ha ${ }^{1} \cdot \mathrm{JiSun}^{\mathrm{Lee}}{ }^{2} \cdot$ MinHee Kim $^{2} \cdot \mathrm{HyeYoung}$ Son $^{2}$. \\ GiYong Lee ${ }^{2} \cdot$ Ravi Gautam ${ }^{3} \cdot$ Yong Heo $^{1,2,3}$ (1)
}

Published online: 9 November 2021

(c) Korean Society of Toxicology 2021

\section{Correction to: Toxicol Res (2021) 37:443-450 https://doi.org/10.1007/s43188-020-00086-7}

In the Acknowledgements section of this article the funding information was incorrect. It should have read 'This research was supported by the Risk Assessment Program for Management of Microplastics Project, funded by Korea Ministry of Environment (MOE) (grant No. 2020003120002) and the educational training program for the management of information on the hazards and risk of chemical substances funded by the Ministry of Environment, Korea (entrusted to Korea Chemicals Management Association). This study was conducted as a part of the first author's master's thesis.'

The original article can be found online at https://doi.org/10.1007/ s43188-020-00086-7.

Yong Heo

yheo@cu.ac.kr

1 Department of Toxicity Assessment, The Graduate School of Medical and Health Industry, Daegu Catholic University, Gyeongsan, Korea

2 GLP Center, Daegu Catholic University, Gyeongsan, Korea

3 Department of Occupational Health, College of Bio and Medical Sciences, Daegu Catholic University, 13-13 Hayang-ro, Gyeongsan 38430, Korea 